\title{
Culture Shapes the Distinctiveness of Posed and Spontaneous Facial Expressions of
}

\section{Anger and Disgust}

\author{
Xia Fang ${ }^{1}$, Disa A. Sauter ${ }^{2}$, Marc W. Heerdink ${ }^{2}$, \& Gerben A. van Kleef ${ }^{2}$ \\ ${ }^{1}$ Zhejiang University \\ ${ }^{2}$ University of Amsterdam
}

Correspondence concerning this article should be addressed to Xia Fang, Department of Psychology and Behavioral Science, Zhejiang University, 148 Tianmushan Road, Hangzhou, China, e-mail: emma.fang88@gmail.com. Contact information for other authors: Disa Sauter, D.A.Sauter@uva.nl; Marc Heerdink, M.W.Heerdink@uva.nl; Gerben van Kleef, G.A.vanKleef@uva.nl.

Author Contributions: X. Fang, D. A. Sauter, and G. A. Van Kleef developed the study concept and design. X. Fang collected data. X. Fang and M. W. Heerdink performed the data analysis and interpretation under the supervision of D. A. Sauter and G. A. Van Kleef. X. Fang drafted the manuscript, and M. W. Heerdink, D. A. Sauter, and G. A. Van Kleef provided critical revisions. All authors approved the final version of the manuscript for submission.

Funding: This research was facilitated by a scholarship from the China Scholarship Council awarded to the first author, and grants from the Netherlands Organisation for Scientific Research (NWO) awarded to the second (275-70-033) and last (452-09-010) authors.

Conflict of Interest: The authors declare that they have no conflict of interest. 


\begin{abstract}
There is a growing consensus that culture influences the perception of facial expressions of emotion. However, little is known about whether and how culture shapes the production of emotional facial expressions, and even less so about whether culture differentially shapes the production of posed versus spontaneous expressions. Drawing on prior work on cultural differences in emotional communication, we tested the prediction that people from the Netherlands (a historically heterogeneous culture where people are prone to low-context communication) produce facial expressions that are more distinct across emotions compared to people from China (a historically homogeneous culture where people are prone to highcontext communication). Furthermore, we examined whether the degree of distinctiveness varies across posed and spontaneous expressions. Dutch and Chinese participants were instructed to either pose facial expressions of anger and disgust, or to share autobiographical events that elicited spontaneous expressions of anger or disgust. Using the complementary approaches of supervised machine learning and information-theoretic analysis of facial muscle movements, we show that posed and spontaneous facial expressions of anger and disgust were more distinct when produced by Dutch compared to Chinese participants. These findings shed new light on the role of culture in emotional communication by demonstrating, for the first time, effects on the distinctiveness of production of facial expressions.
\end{abstract}

Keywords: culture, facial expressions, emotion, posed, spontaneous 


\section{Culture Shapes the Distinctiveness of Posed and Spontaneous Facial Expressions of}

\section{Anger and Disgust}

Emotional communication via facial expressions rests on the production (encoding) and perception (decoding) of emotional expressions (Scherer, Clark-Polner, \& Mortillaro, 2011; Van Kleef, 2016). The extent to which emotional facial communication is universal versus culturally specific is a contentious issue. The vast majority of research on cultural influences in emotional facial communication to date has focused on emotion perception the act of inferring an expresser's emotion based on physical properties of the expression. Early research suggested that the perception of expressions of at least some emotions is highly similar across cultures, such that people from different cultures perceive similar emotions from facial expressions (e.g., Ekman, 1973; Izard, 1994). More recent research has uncovered cultural differences in patterns of emotion perception (Elfenbein \& Ambady, 2002), including in the degree to which people perceive mixed emotions in expressions (Fang et al., 2018, 2019; Kayyal \& Russell, 2013), suggesting that there are differences in how people from different cultures interpret emotional facial expressions.

In contrast to the substantial literature on cultural influences on emotion perception, surprisingly little research has examined the extent to which culture shapes the production of emotional facial expressions - the act of expressing a particular emotion by producing physical cues in the face. Moreover, the work that has been conducted on emotion production has focused primarily on posed expressions - deliberately showing an emotion in order to attempt to convey it (e.g., Elfenbein et al., 2007). Little research has examined spontaneous expressions that occur involuntarily and naturally during interactions (Hess et al., 2016; Schaefer et al., 2010). Here, we sought to investigate for the first time how culture shapes the distinctiveness of both posed and spontaneous facial expressions.

\section{Posed versus Spontaneous Facial Expressions Across Cultures}


Previous research has uncovered differences between posed and spontaneous expressions (Hunt, 1941; Matsumoto et al., 2009; Matsumoto \& Willingham, 2009). It has been proposed that posed facial expressions lack facial muscle movements that do occur in spontaneous expressions and/or contain extraneous facial muscle movements that do not occur in spontaneous expressions (Matsumoto et al., 2009). Empirical work based on Western samples has shown that posed expressions are more intense and prototypical than spontaneous expressions (Tcherkassof et al., 2007). However, no study has compared the production of posed and spontaneous facial expressions in a cross-cultural setting. The extent to which culture influences posed and spontaneous expressions is thus unclear.

Studies of the facial expressions of blind individuals provide some suggestive evidence that posed expressions may be affected by culture to a higher degree than spontaneous expressions. Early observational studies found that congenitally blind individuals produced similar spontaneous facial expressions to those of sighted individuals, while considerable differences between blind and sighted individuals were found for posed expressions (Freedman, 1964; Thompson, 1941). Those results are consistent with later studies that employed standardized measurements of facial muscle movements (Galati et al., 2001; Matsumoto \& Willingham, 2009; Rinn, 1991). It has been argued that the differences between posed and spontaneous facial expressions may reflect differential effects of learning processes. Specifically, posed expressions may be affected by social learning of culturally shaped prototypes to a greater degree than spontaneous expressions (Matsumoto \& Willingham, 2009; Rinn, 1991).

However, empirical studies of the recognition of emotional expressions suggest that culture-specific learning processes can also shape spontaneous expressions (Crivelli et al., 2017; Kayyal \& Russell, 2013; Naab \& Russell, 2007; Nelson \& Russell, 2013). For instance, when classifying spontaneous facial expressions produced by individuals from Papua New 
Guinea, fewer than $30 \%$ of participants from America and Palestine selected the predicted emotion label (Kayyal \& Russell, 2013). Even for other residents from Papua New Guinea, only between $13 \%$ and $38 \%$ of respondents selected the predicted emotion label (Crivelli et al., 2017). This agreement is far below 70-90\%, a criterion previously used to support the claim of universality of facial expressions of emotion (Haidt \& Keltner, 1999). Other studies have also documented that the recognition of spontaneous facial expressions varies across emotions as well as cultures (Kayyal \& Russell, 2013; Matsumoto et al., 2009). Past research on emotion perception thus points to cross-cultural variability in spontaneous facial expressions.

Although the perception and production of emotion are different processes, they are both part and parcel of the process of communicating emotions (Scherer, Clark-Polner, \& Mortillaro, 2011). We thus expected that evidence from both processes (perception and production) would shed light on the role of culture in emotional facial communication. While the limited evidence on the production of emotional facial expressions seems to suggest that culture has a small, or even negligible, impact on spontaneous compared to posed expressions, evidence from studies on emotion perception points to a role for culture in the perception of spontaneous facial expressions. We thus included both posed and spontaneous expressions in the same study and examined how culture shapes the production of posed versus spontaneous facial expressions.

\section{Emotional Facial Communication Across Cultures}

Individuals from different cultures differ in how they communicate with each other, driven in part by the degree of historical heterogeneity in a society (Gudykunst et al., 1996; Hall, 1976; Niedenthal et al., 2019). Populations of historically homogeneous societies (including countries such as China and Japan) originate from one or a few source countries. They have common values and beliefs (Mesquita \& Frijda, 1992), and are usually 
characterized by stable and predictable interpersonal relationships. In historically homogeneous societies, a lot of information is conveyed by the physical context in conjunction with a shared understanding of reality, and comparatively less information is embedded in the explicit part of the message, a style referred to as "high-context communication" (Hall, 1976). Consequently, communication in homogeneous societies is more indirect and ambiguous (Gudykunst \& Ting-Toomey, 1988). In contrast, historically heterogeneous societies (including countries such as the United States and the Netherlands) have populations who originate from many source countries, and contain diverse values and beliefs brought in from the different cultures of origin. To navigate such diverse social worlds, individuals need to convey their feelings and intentions directly and precisely, a style referred to as "low-context communication" (Hall, 1976). The communication in heterogenous societies is thus more direct and specific (Gudykunst \& Ting-Toomey, 1988).

This cultural difference in communication styles might also characterize the process of emotional facial communication. Because a lot of information is embedded in the context in high-context communication cultures, we expected that a facial expression on its own is less likely to contain sufficient information to allow observers to identify an underlying emotional state. In contrast, in cultural groups characterized by a low-context communication style, emotional facial expressions would be expected to contain all the information needed to identify the underlying emotional state. Individuals from historically heterogeneous cultures would thus be expected to produce more distinct emotional expressions than individuals from homogeneous cultures, that is, their facial expressions would contain more elements that are specific to the emotion at hand (as opposed to elements that are shared with other emotions), which would help observers identify the underlying emotional state and distinguish it from other emotional states. 
Two studies provide suggestive evidence consistent with this possibility. Wood and colleagues (2016) reanalyzed the results of 92 studies reporting a total of 212 average crosscultural emotion recognition scores and found that individuals from historically heterogeneous cultures produce facial expressions of emotion that are recognized more accurately than expressions produced by individuals from homogeneous cultures. In another study, Rychlowska and colleagues (2015) showed that people from heterogeneous countries believe that they should freely express or even amplify emotional expressions, whereas people from homogeneous countries tend to think they should dissimulate emotional expressions. These arguments are further bolstered by a recent review on historical heterogeneity and emotion communication that suggests that historically heterogeneous societies promote expressivity and clarity in emotional expressions (Niedenthal et al., 2019).

These findings provide novel insights into how culture might influence the distinctiveness of emotional expressions. However, these studies examined emotion production by analyzing perceptual judgments of expressions or subjective reports of social norms. Arguably the most direct and valid way of examining facial expressions produced by individuals from homogeneous versus heterogeneous cultures is to measure their actual patterns of facial expressions of emotions. Therefore, in the present study, we recruited Dutch and Chinese individuals, who are from historically heterogeneous and homogeneous cultures respectively (Niedenthal et al., 2019; Rychlowska et al., 2015; Wood et al., 2016), and analyzed their facial muscle movements as they produced posed or spontaneous facial expressions of emotions.

Here, we focused on facial expressions of two emotions: anger and disgust. These emotions were selected based on three reasons. First, anger and disgust are widely held to be basic emotions (Ekman, 1992; Izard, 2007) and would thus be expected to occur in both Chinese and Dutch cultures. Second, although anger and disgust are distinct emotions, they 
are conceptually related (Giner-Sorolla et al., 2018) and associated with morphologically similar facial configurations (Cordaro et al., 2018; Susskind et al., 2007). Examining emotional expressions that show partial overlap in their facial morphology (rather than expressions that are entirely distinct, e.g., anger and happiness) allows for a meaningful test of the effects of culture on expressive distinctiveness (effects of culture on the distinctiveness of emotion expression, if they exist, are unlikely to manifest in comparisons between anger and happiness). Third, recent studies have found that Westerners are more likely to perceive specific emotions in facial expressions of anger and disgust, whereas Easterners tend to perceive mixed emotions in these facial expressions (Fang et al., 2018, 2019). We predicted that this inclination may also become manifest in the production of facial expressions of anger and disgust, with Westerners' facial expressions of anger and disgust being more distinct than Easterners' facial expressions of these emotions. Examining anger and disgust thus allows for direct comparisons of cultural influences on emotion production that were to be tested in the current research with cultural influences on emotion perception observed in previous work (Fang et al., 2018, 2019).

\section{The Present Research}

Based on prior work on cultural differences in the distinctiveness of emotional communication, we expected that facial configurations of anger and disgust produced by Dutch people would be more distinct than those produced by Chinese people. In a betweensubjects design, Dutch and Chinese participants were asked to either pose facial expressions of anger and disgust with the goal of being understood by their friends (i.e., posed facial expressions), or to share autobiographical events that had elicited feelings of anger or disgust (i.e., spontaneous facial expressions).

In order to test the distinctiveness of emotional expressions across cultures, we first Facial Action Coding System (FACS; Ekman et al., 2002) coded all facial expressions 
and then conducted two sets of analyses on the coded facial action units (AUs) of the facial expressions. Specifically, the first set of analyses were conducted at the $A U$ level. We adapted Chen and colleagues' (2018) approach based on Mutual Information (MI) and Monte Carlo simulation to identify AUs that are specific to facial expressions of anger or disgust depending on culture and spontaneity. If facial expressions of anger and disgust are more distinct when produced by Dutch as compared to Chinese participants, a larger number of specific (non-overlapping) AUs should be expected in Dutch expressions than Chinese expressions. The second set of analyses were conducted at the expression level. Given prior theorizing on cultural differences in expressive distinctiveness, a completely naïve observer (who has no knowledge about prototypes of emotional expressions and no cultural background) should find it easier to distinguish between facial expressions of anger and disgust produced by Dutch as compared to Chinese participants. As an analogy to this naïve observer, we used a machine learning approach to categorize expressions based on the patterns of facial AUs. Our hypothesis would be supported if the machine learning model is more accurate in categorizing facial expressions of Dutch versus Chinese participants. Across these analytical approaches, we examined whether cultural differences in the distinctiveness of emotion production would be consistent across posed and spontaneous facial expressions.

\section{Method}

\section{Participants and Design}

In the absence of studies examining similar research questions, sample size was determined a priori based on previous cross-cultural studies of emotion production (21-25 participants per culture by Cordaro et al., 2018; 30 participants per culture by Elfenbein et al., 2007), as well as feasibility, given the time-consuming nature of manually coding facial AUs. We sought to recruit 50 participants from each culture to produce posed or spontaneous facial expressions. We employed a 2 (Culture: Chinese, Dutch $) \times 2$ (Emotion: Anger, Disgust $) \times 2$ 
(Spontaneity: Posed, Spontaneous) mixed design, with Emotion being a within-subjects factor and Culture and Spontaneity being between-subjects factors. To ensure that participants from China and the Netherlands would be similar in age and socioeconomic and educational background, we recruited student samples from the two countries.

Ninety-nine Dutch participants were recruited from a university in the Netherlands, and 97 Chinese participants were recruited from a university in China. Thirty-two participants were excluded from the analysis, either because their faces were not entirely visible or because no facial movement occurred in the specified frame for facial expression coding (see below). The majority of the excluded participants were from the spontaneous condition $(N=27)$, because participants in the spontaneous condition were not explicitly asked to look at the camera or to show emotional expressions. This resulted in 49 Dutch $\left(M_{\text {age }}\right.$ $=22.94, S D=3.00 ; 13$ men $)$ and 45 Chinese $\left(M_{\mathrm{age}}=19.04, S D=1.33 ; 23\right.$ men $)$ participants in the posed condition, and 35 Dutch $\left(M_{\mathrm{age}}=23.20, S D=3.05 ; 11 \mathrm{men}\right)$ and 35 Chinese $\left(M_{\mathrm{age}}\right.$ $=19.49, S D=1.54 ; 11$ men) participants in the spontaneous condition. In return for participation, Dutch participants received 0.5 course credits, and Chinese participants received $20 \mathrm{CNY}$ (approximately $4 \mathrm{USD}$ ).

\section{Procedure}

All instructions and materials were translated from English into Chinese and Dutch by means of the standard translation/back-translation procedure. Participants were randomly assigned to the posed or spontaneous condition. In each condition, participants started with a neutral condition to get familiarized with the procedure; the order of anger and disgust was counterbalanced between participants. All measures, manipulations and exclusions are reported.

\section{Posed Condition}


In the posed condition, participants were asked to produce facial emotional expressions such that "their friends would be able to understand easily what they feel". This instruction has previously been employed as a way to elicit voluntarily facial expressions (Elfenbein et al., 2007). Before each pose, participants were presented with an emotion label, a definition of the emotion term, and a one-sentence emotion story drawn from previous cross-cultural studies (Ekman \& Cordaro, 2011; Matsumoto et al., 2005; see Tables S1 in the Supplementary Material for details). This sought to ensure that participants from different cultures had a similar understanding of the target emotion terms (e.g., Cordaro et al., 2018; Sauter et al., 2010). Participants tested various expressions with a video camera. Once they were satisfied with their pose, participants rated the extent to which they had experienced each of five emotions (anger, disgust, fear, sadness, and happiness) while posing, using scales between 0 and $10(0=$ not at all, $10=$ extremely $)$. Participants also indicated how difficult they found it to pose each target emotion on a scale from 0 to $10(0=$ very easy, $10=$ very difficult). Having completing the ratings after posing the first emotion, participants pressed a button that rang a bell, upon which the experimenter provided them with the next emotion label, definition, and example situation, and the procedure was repeated. The experimenter was not in the room with the participant during the posing and did not provide feedback or recommendations of any kind. The entire posing session was filmed.

After producing expressions of anger and disgust, participants were led to another room, where they looked at the video of their own expressions. They were asked to choose the frame that they thought represented the clearest expression of the instructed emotion. This approach ensures that the selected frame is an accurate representation of the participant's intended expression and avoids introducing experimenter bias into the coding procedure. Once they had selecting the frame, participants rated the clarity and intensity of their 
expression in that frame using scales of 0 to $10(0=$ not at all clear/intense, $10=$ extremely clear/intense).

\section{Spontaneous Condition}

In the spontaneous condition, we used a cover story telling participants that they were going to help develop a robot that can understand human emotions. Participants were instructed to take their time to recall a past life event that involves angry or disgusted emotional experiences and tell this past experience in detail to the robot, Eva (see Eliciting Spontaneous Emotional Facial Expressions in the Supplementary Material). This relivedemotion method has been shown to be an effective means of eliciting emotional expressions in previous studies (e.g., Tsai \& Chentsova-Dutton, 2003). We used a robot instead of a human being because of the concern that (especially Chinese) participants might feel uncomfortable sharing negative life events with strangers and may therefore de-amplify or even mask their expressions (e.g., Matsumoto et al., 2008). Previous research has shown that interacting with virtual humans can make people more willing to disclose negative affective information (Lucas et al., 2014).

After sharing each story, participants indicated how comfortable they felt sharing their experience with Eva on a scale from 0 to $10(0=$ very uncomfortable, $10=$ very comfortable). The rest of the procedure was identical to the posed condition. Participants were fully debriefed upon completing the experiment.

\section{Results}

All anonymized raw data are available at https://osf.io/x7dbt/?view_only=386e7b6536164130adeede30708b3504.

\section{Preliminary Analyses}

In both the posed and spontaneous conditions, participants' self-reported judgments of their emotional expressions and experiences were included to test whether any cultural 
differences found in facial muscle movements could be due to cultural differences in selfreports on expressions and experiences.

\section{Self-reports on Expressions}

Using the self-report ratings, we tested whether Dutch and Chinese participants differed in their ratings of their own expression clarity, expression intensity, difficulty of posing expressions, and how comfortable they felt sharing autobiographical events with the robot. Whereas Chinese participants gave higher ratings than Dutch participants on clarity $\left(M_{\text {Chinese }}=7.43, S D=1.45 ; M_{\text {Dutch }}=6.73, S D=1.56\right)$, intensity $\left(M_{\text {Chinese }}=6.89, S D=1.43\right.$; $\left.M_{\text {Dutch }}=6.34, S D=1.67\right)$, and comfort $\left(M_{\text {Chinese }}=6.13, S D=2.17 ; M_{\text {Dutch }}=5.00, S D=2.08\right)$, $p s<.030$ (see Table S2 in the Supplementary Material), the groups did not differ in experienced difficulty in posing expressions $\left(M_{\text {Chinese }}=4.71, S D=1.86 ; M_{\text {Dutch }}=4.99, S D=\right.$ $1.84), F(1,92)=0.53, p=.467, \eta_{p}^{2}=.006$. These results suggest that if the hypothesized lower distinctiveness in Chinese participants' facial expressions were to be observed, it could not be explained by their greater difficulty in posing expressions or being more uncomfortable in sharing negative autobiographical events, or by lower clarity or intensity in the judgments of their own expressions.

\section{Self-reports on Experiences}

We first sought to establish whether participants experienced the emotions that they were asked to pose or share. In both posed and spontaneous conditions and in both of the cultural groups, participants rated their emotional experiences higher on the target emotion scale (e.g., anger ratings for anger expressions) than all nontarget emotion scales (disgust, fear, sadness, and happiness ratings for anger expressions), $p \mathrm{~s}<.001$ (see Table S3 in the Supplementary Material). These results establish that participants indeed experienced the target emotions. 
Second, we tested whether the induced emotional experiences were comparable across the two cultural groups, focusing on the target emotion and the nontarget emotion that has been found to be most confusable with the target one, that is, the ratings of anger versus disgust. While Chinese participants $(M=5.53, S D=1.89)$ experienced more intense emotions than did Dutch participants $(M=4.18, S D=1.69), F(1,160)=23.58, p<.001, \eta_{\mathrm{p}}^{2}=.128$, the groups did not differ in the degree to which they experienced mixed emotions ( $M$ |anger-

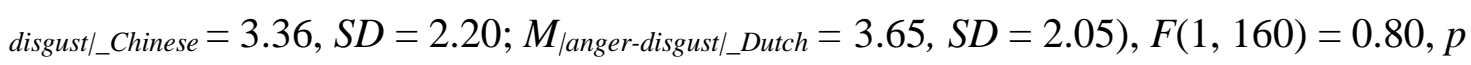
$=.372, \eta_{\mathrm{p}}^{2}=.005$ (see Table S4 in the Supplementary Material). Therefore, we found no evidence that Chinese participants experienced more mixed emotions than Dutch participants in the current paradigm. It thus rules out the possibility that any observed cultural differences in distinctiveness between anger and disgust expressions could be explained by cultural differences in mixed emotional experiences.

\section{Main Analyses}

\section{AU Activity in Expressions of Anger and Disgust}

The facial expressions were coded by FACS, with all facial AUs being dummy coded for absence or presence of activation for each expression. A certified FACS coder coded all the expressions, and a second certified FACS coder coded $20 \%$ of the expressions. The agreement level between the two coders was 63\%, which is deemed acceptable (Ekman et al., 2002). The first coder's classifications were used for the analyses.

After excluding AUs that were never active in any condition, there were a total of 40 facial AUs to consider in the analysis. The activation of all AUs in each of the eight conditions $($ Emotion $\times$ Spontaneity $\times$ Culture $)$ is displayed in Figure 1 . Most of the AUs were rarely active, and only a few AUs were active in a substantial proportion of participants. To distinguish meaningful AU activity from random activity, we implemented the Monte Carlo 
simulation method developed by Chen and colleagues (2018) to identify the AUs that were most frequently active in each condition.

To illustrate the use of a Monte Carlo simulation method to identify highly frequent AUs for each condition, consider, for example, identifying the AUs that are highly frequent across the 49 posed Dutch facial expressions of anger. First, for each of the 40 AUs, we computed the proportion of the 49 expressions in which the AU was active. For example, we observed brow lowerer (AU4) in 44 expressions, resulting in a 44/49 proportion of AU4 activation. Second, to determine which proportions could be considered highly frequent, we performed a Monte Carlo simulation with 5,000 iterations. In each iteration, we filled a new matrix of 49 expressions $\times 40$ AUs by randomly sampling AU activation from the original pattern of AU activity (a total of 141 AUs were active). For instance, one random iteration might produce a frequency of 7/49 for AU1, 1/49 for AU4, and so forth. Combining the resulting proportions of AU activity across all matrices (i.e., 40 proportions per iteration, and $40 \times 5,000=200,000$ proportions in total) yields the distribution of AU frequencies that would be observed if AU activities were completely random. This (null) distribution was then used to test whether the observed $44 / 49$ proportion of brow lowerer (AU4) in the posed Dutch expressions of anger is significantly higher than chance, calculated as it being above the 95th percentile of the generated distribution (one-tailed $\mathrm{p}<.05$ ). If so, brow lowerer (AU4) would be considered a highly frequent AU in this condition. We repeated this procedure for each AU and for each condition (Emotion $\times$ Spontaneity $\times$ Culture) separately, thereby taking into account that conditions differed in the total number of active AUs. The AUs that we identified as highly frequent are indicated with red crosses in Figure 1. 


\section{Figure 1}

The Activation of 40 AUs in Each of the Eight Conditions (Emotion $\times$ Spontaneity $\times$ Culture)

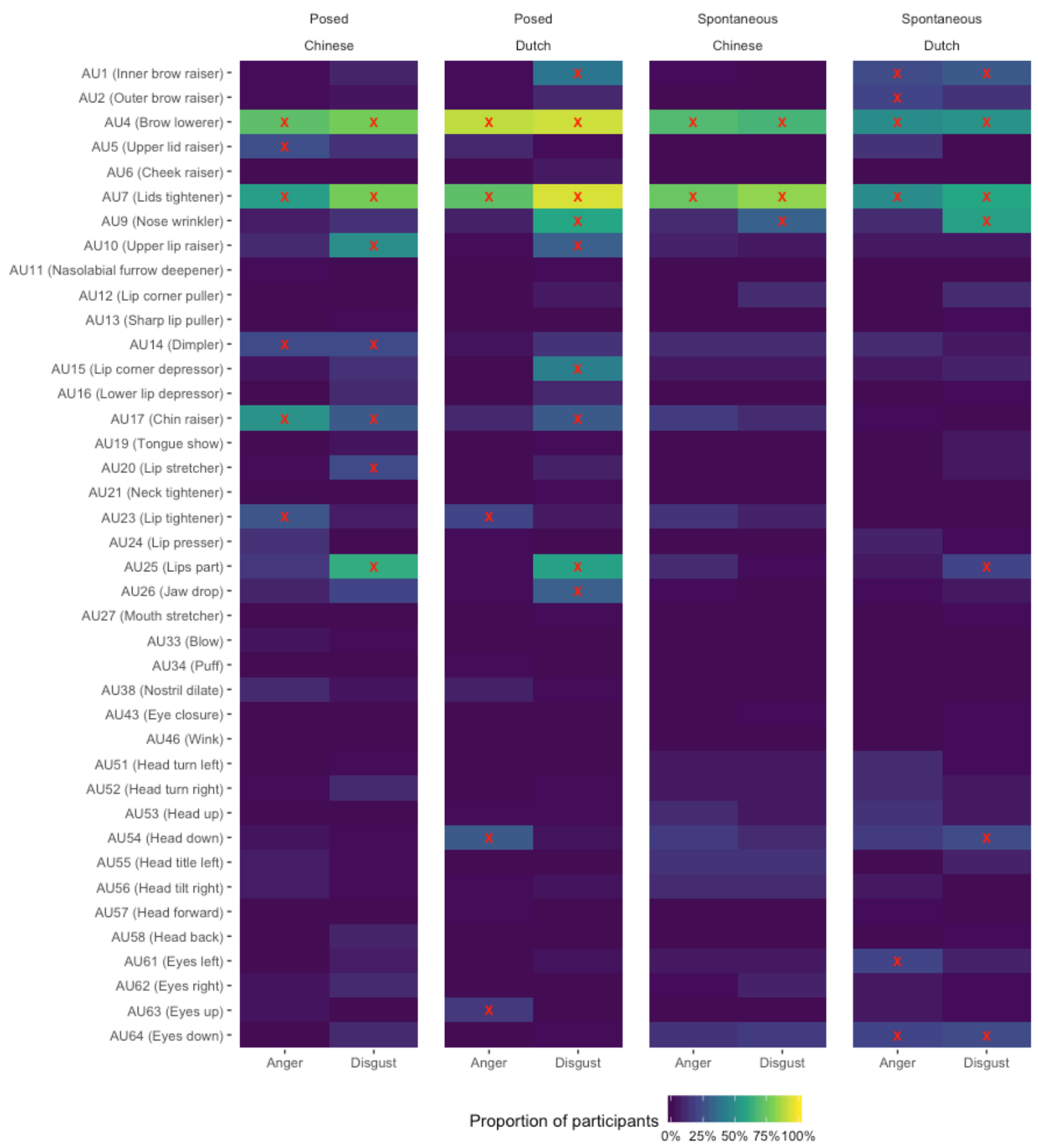

Note. For each condition, the color-coded matrix shows the proportion of participants activating each AU (labels on the left). Warmer colors indicate more participants; cooler colors indicate fewer participants (see color bar at the bottom). The red crosses indicate frequent AUs for that condition, as calculated using a Monte Carlo simulation. 


\section{Specific AUs in Expressions of Anger and Disgust Across Cultures and Spontaneity}

To identify AUs that are specific to facial expressions of anger or disgust depending on culture and spontaneity, we adapted Chen and colleagues' (2018) approach based on Mutual Information (MI) and Monte Carlo simulation. MI reflects how much "knowledge" one can gain of emotion categories (here, anger versus disgust) by knowing the other variable (here, the absence versus presence of a given AU). We conducted our analysis only with the frequent AUs (indicated by red crosses in Figure 1) to ensure that the specific AUs identified are representative of the expressions of anger and disgust. Specifically, we randomly reshuffled the emotion categories 1,000 times for each cell of the design (Culture $\times$ Spontaneity). At each iteration, we calculated the MIs between the frequent AUs and the (reshuffled) emotion categories, and retained the maximum MI. This yields a distribution of MI values that reflects completely random associations between AUs and emotion categories. Then, we computed the MI between each of the 18 frequent AUs (indicated with red crosses in Figure 1) and the actual emotion categories (anger or disgust). These MI values are deemed significant if they exceed the $95^{\text {th }}$ percentile of the random distribution of MI values.

The results showed that the number of specific AUs (i.e., AUs with significant MI values; see Figure S1 in the Supplementary Material for details) differed depending on culture and spontaneity, ranging from nine in the posed Dutch condition, to zero in the spontaneous Chinese condition. As predicted, more AUs were emotion-specific in the expressions produced by Dutch as compared to Chinese participants. Moreover, more AUs were emotion-specific in the posed as compared to the spontaneous expressions. Looking at specific AUs, for the posed expressions of Dutch participants, seven AUs (AUs 1 [inner brow raiser], 7 [lids tightener], 9 [nose wrinkler], 10 [upper lip raiser], 15 [lip corner depressor], 25 [lips part], and 26 [jaw drop]) were specific to the expressions of disgust, and two (AUs 54 [head down] and 63 [eyes up]) were specific to the expressions of anger. For the posed 
expressions of Chinese participants, three AUs (AUs 10 [upper lip raiser], 20 [lip stretcher] and 25 [lips part]) were specific to the expressions of disgust, but no AU was specific to the expressions of anger. For the spontaneous expressions of Dutch participants, AU9 (nose wrinkler) was specific to the expressions of disgust, but no AU was specific to the angry expressions. For the spontaneous expressions of Chinese participants, no AUs were found to be specific to either expressions of anger or disgust. Together, these findings provide AUbased evidence that the posed and spontaneous expressions produced by Dutch participants were more distinct than those produced by Chinese participants.

The Monte Carlo approach identifies the frequent AUs for the angry and disgusted expressions in a given condition, and the combined approach of Mutual Information and Monte Carlo simulation identifies the AUs that are specific to the angry or disgusted expressions in a given condition. These results do not, however, necessarily indicate that the combination of frequent or specific AUs in a given condition makes up the prototypical expression in that condition. To take an extreme example, it is possible that in a given condition, both AU1 (inner brow raiser) and AU7 (lids tightener) are identified as specific AUs to disgust, even though they are observed in mutually exclusive halves of the data, and thus never occur together. This method thus does not allow for comparing the distinctiveness between combinations of AUs for angry and disgusted expressions. To complement this approach, we therefore conducted a second set of analyses at the level of combinations of AUs (i.e., at the expression level). Specifically, we implemented a machine learning approach (aka "a naïve observer") to categorize expressions based on the distinctiveness of the AU patterns between angry and disgusted expressions. Our prediction would be supported by the machine learning model being more accurate in categorizing the expressions of Dutch as compared to Chinese participants.

\section{Machine Learning-Based Facial Expression Categorization}


To apply a supervised machine learning approach, we first 'trained' models with a series of labeled (i.e., pre-classified) angry and disgusted expressions with their features (AU Activity, Culture, and Spontaneity); then, the models were used to categorize expressions (without being given labels) as anger or disgust. Specifically, we used logic regression with the LogicReg package in R (Kooperberg \& Ruczinski, 2018) to differentiate between angry and disgusted expressions. We first fit models with varying numbers of "trees" (or predictors, ranging from one to three) and varying numbers of "leaves" (or features, ranging from two to the maximum number, thirteen) per tree (Ruczinski et al., 2003). These parameters allow for anything between the simplest possible model (here: one predictor combining two AUs) and a model that combines nearly all of the observed 40 AUs in some way (here: three trees combining 13 AUs each allow for using a total of 39 AUs). To prevent overfitting the data (i.e., the model learning idiosyncratic patterns rather than true regularities), we used repeated $\mathrm{k}$-fold cross-validation (20 folds, 20 repeats) to find the parameters to yield the best generalizing model (cross-validated Accuracy $=77.3 \% ; \kappa=0.55$ ). Applying these rules to the full data set yielded correct predictions in $78.4 \%$ of the cases. The similarity of this percentage to the cross-validated accuracy, $77.3 \%$, indicates that the amount of overfitting is likely to be limited.

Based on the categorization accuracy obtained from the logic regression, we conducted our hypothesis test using a logistic mixed-effect model with the blme package in $\mathrm{R}$ (Chung et al., 2013). Accuracy was regressed on Culture, Spontaneity, and Emotion, and a random intercept for participant was included to partially control for individual differences in expressivity. The three-way interaction was not significant $(p=.553)$, nor were the Emotion $\times$ Spontaneity $(p=.098)$ or Culture $\times$ Spontaneity $(p=.215)$ two-way interactions. The Emotion $\times$ Culture interaction $(p=.028)$ and all main effects were significant $(p s<.010)$. As predicted, accuracy was generally higher for the expressions of Dutch participants than those 
of Chinese participants $(p=.001)$. This effect was further qualified by Emotion $(p=.028)$, with cultural differences being greater for expressions of disgust $(O R=0.16, p<.001)$ than for expressions of anger $(O R=0.65, p=.342)$ (see in Table 1). Furthermore, accuracy was higher for posed than for spontaneous expressions $(O R=2.36, p=.009)$. Finally, accuracy was higher for angry than disgusted expressions $(p=.010)$ although simple effects analyses showed that this was only the case among Chinese $(O R=3.46, p=.001)$, but not among Dutch $(O R=0.79, p=.660)$ participants. These results indicate that Chinese participants' disgusted expressions were more often miscategorized as anger compared to Dutch participants' disgusted expressions, suggesting that Chinese participants' disgusted expressions were less distinct from their angry expressions. These findings thus provide expression-based evidence that facial expressions of anger and disgust were more distinct in Dutch as compared to Chinese participants, and this pattern of results did not differ between posed and spontaneous expressions.

\section{Table 1}

Machine Learning Categorization Accuracy for Each of the Eight Conditions (Emotion $\times$ Spontaneity $\times$ Culture)

\begin{tabular}{ccccc}
\hline Culture & Spontaneity & $\boldsymbol{N}$ & Anger & Disgust \\
\hline \multirow{2}{*}{ Chinese } & Posed & 45 & $82.2 \%$ & $66.7 \%$ \\
& Spontaneous & 35 & $80.0 \%$ & $45.7 \%$ \\
\multirow{2}{*}{ Dutch } & Posed & 49 & $89.8 \%$ & $95.9 \%$ \\
& Spontaneous & 35 & $82.9 \%$ & $74.3 \%$ \\
\hline
\end{tabular}

\section{Intra-individual versus Inter-individual Variation in Emotional Expression}

Why was the categorization of Chinese expressions more challenging than that of Dutch expressions? One possibility is that it reflects greater intra-individual similarities between expressions of these two emotions in the Chinese sample. That is, individual 
Chinese participants might have used similar facial movements to express anger and disgust. As a result, the machine learning model (aka "a naïve observer") found it more difficult to distinguish between the angry and disgusted expressions of Chinese compared with Dutch participants, resulting in lower distinctiveness in Chinese expressions. Another possibility is that it was caused by larger inter-individual variations in Chinese expressions of these two emotions. If so, there would be a larger array of facial prototypes for anger and disgust in the Chinese sample, and the machine learning model would consequently have found it more challenging to distinguish between expressions of anger and disgust produced by Chinese as compared to Dutch participants.

To test which of these accounts better fit the data, we used Hamming distance to measure the dissimilarity between facial expressions of anger and disgust for each individual across all 40 AUs. Specifically, a 2 Culture $\times 2$ Spontaneity ANOVA of these distances (with Type III errors) revealed a main effect of Spontaneity, $F(1,160)=15.90, p<.001, \eta_{\mathrm{p}}^{2}=0.09$, and a two-way interaction, $F(1,160)=6.13, p=.014, \eta_{\mathrm{p}}^{2}=0.04$. Simple effects analyses showed that Chinese participants had a smaller Hamming distance than Dutch participants for spontaneous expressions, $t(160)=3.36, p=.002$, but not for posed expressions, $t(160)=$ $0.10, p>.999$. This indicates that the spontaneous Chinese expressions of anger and disgust had larger intra-individual similarities of facial movements than the spontaneous Dutch expressions, whereas no cultural difference was found for the posed expressions. These results suggest that the lower distinctiveness of the spontaneous Chinese expressions is likely at least in part due to individual Chinese participants using more similar facial movements to express anger and disgust. However, the lower distinctiveness of the posed Chinese expressions appears to reflect a larger variation in prototypes for posed facial expressions of anger and disgust in Chinese relative to Dutch culture.

\section{Discussion}


The present research examined whether Dutch versus Chinese people differ in the distinctiveness of posed and spontaneous emotional facial expressions. Using a combined Mutual Information and Monte Carlo simulation approach, we found more emotion-specific AUs in both posed and spontaneous expressions produced by Dutch as compared to Chinese participants. Using a machine learning approach, we found that Dutch participants' expressions were better categorized than Chinese participants', regardless of the spontaneity of expressions. These two distinct approaches - one at the AU level and one at the expression level — provide convergent evidence that the posed and spontaneous expressions of anger and disgust produced by Dutch participants were more distinct as compared to the expressions by Chinese participants.

\section{Posed versus Spontaneous Facial Expressions}

The current results demonstrate that facial expressions of anger and disgust were more distinct when they were posed as compared to spontaneous: The posed expressions of anger and disgust had more emotion-specific AUs compared with the spontaneous ones, and the posed expressions of anger and disgust were better categorized than the spontaneous expressions. Two possibilities may account for such differences between posed and spontaneous expressions. First, participants reported more mixed feelings in the spontaneous as compared to the posed conditions (see Tables S3 and S4 in the Supplementary Material for details). It is thus possible that the relatively more mixed spontaneous facial expressions were caused by participants experiencing more mixed feelings in the spontaneous condition.

Second, posed and spontaneous facial expressions might be characterized by partly different patterns of facial AUs. Previous research suggests that posed expressions might lack some facial muscle movements that do occur in spontaneous expressions or may contain extraneous facial muscle movements that do not occur in spontaneous expressions (Matsumoto et al., 2009). Our data are consistent with this possibility: Posed and spontaneous 
emotional expressions shared a number of frequent AUs, yet they were also characterized by frequent AUs that did not overlap between posed and spontaneous expressions (see red crosses in Figure 1).

\section{Chinese versus Dutch Participants’ Facial Expressions}

Consistent with previous research on the production of facial expressions across cultures (Cordaro et al., 2018; Elfenbein et al., 2007), the current results suggest an intermediate position on the universality of emotional facial expressions, such that there are both universal and culturally specific features in expressions of emotion (Cordaro et al., 2018; Elfenbein et al., 2007). For example, the posed angry expressions of Chinese and Dutch participants shared three frequent AUs (AUs 4 [brows lowerer], 7 [lids tightener], and 23 [lip tightener]) but differed on five other AUs (see Figure 1). Similarly, the posed disgusted expressions of Chinese and Dutch participants shared five frequent AUs (AUs 4 [brows lowerer], 7 [lids tightener], 10 [upper lip raiser], 17 [chin raiser], and 25 [lips part]), but differed on six other AUs (see Figure 1). Notably, all of the common AUs shared by cultures in the present study map onto to the AUs found in other studies on facial expressions of anger and disgust across cultures (Cordaro et al., 2018; Du et al., 2014; Jack et al., 2016).

\section{Limitations and Future Directions}

Some limitations of our study merit discussion. First, due to the time-consuming nature of this study (the recruitment of cross-cultural samples that produced either posed or spontaneous emotional expressions, as well as the manual coding of facial movements), we examined only two cultural groups. Researchers might consider including more cultures along the continuum of historical heterogeneity to probe the relationship between historical heterogeneity and the distinctiveness of emotion production. In addition to expressions of anger and disgust, future work might also examine whether our findings extend to another pair of basic emotions that are morphologically similar, i.e., fear and surprise. 
Second, the gender distribution in the posed condition was not equal across the two cultural groups. However, concerns that the unequal gender distribution influenced cultural differences in the distinctiveness of emotion production are mitigated by the fact that we found a similar pattern of results for spontaneous expressions (with expressions of anger and disgust being more distinct for Dutch compared to Chinese participants), where the proportion of male/female participants was the same across the two cultures. Moreover, we included participants' gender in the generalized mixed effect model that predicted the accuracy of the labelling in the machine-learning analysis. There was no evidence for main effects or any interactions involving Gender (the four-way was $p=.097$ and all other terms including Gender were $p \mathrm{~s}>$.157).

While the present research focused on the patterns of facial movements in static facial expressions, it is unclear whether a similar pattern of results would occur for dynamic facial expressions that we typically encounter in everyday life. In addition to facial movements, real-life expressions have other features such as the onset, peak, and offset latency (Krumhuber \& Manstead, 2013). These dynamic features may help differentiate one emotional expression from another. As such, real-life expressions of anger and disgust may well be less overlapping than our estimates, which are based on static images. We hope future research will examine this possibility. Moreover, given that nonverbal emotion communication is not limited to facial expressions of emotion, it would be worthwhile for future research to establish whether similar patterns can be found in other types of expressions, such as vocal and postural expressions of emotion.

\section{Implications and Conclusion}

The relationship between cultural norms and facial expressions has implications for a range of cultural products. For example, previous research has shown that the affective states that people are taught to value within the cultural context they are a part of ("ideal affect") 
may influence how leaders smile in official photos (Tsai et al., 2016). Similarly, the current finding of cultural differences in the distinctiveness of facial expressions may also have potential implications for social interactions and cultural products such as film, advertising, and artificial intelligence. For instance, Western audiences, who are used to more distinctive and categorical emotional expressions, may be confused by Easterner actors' emotional facial expressions, which are less distinct from each other. Thus, film makers targeting international audiences may benefit from awareness of variability in the distinctiveness of emotion production.

Overall, the present study provides a novel empirical contribution to research on nonverbal emotion communication across cultures. We have shown that Dutch individuals, who are from a historically heterogeneous culture characterized by low-context communication, produce posed and spontaneous expressions of anger and disgust in more distinct ways than Chinese individuals, who are from a historically homogeneous culture characterized by high-context communication. The current findings, together with previous findings on cultural differences in the distinctiveness of emotion perception, enhance understanding of the role of culture in emotion communication by illuminating how culture shapes the distinctiveness of emotional facial communication. 


\section{References}

Chen, C., Crivelli, C., Garrod, O. G., Schyns, P. G., Fernández-Dols, J. M., \& Jack, R. E. (2018). Distinct facial expressions represent pain and pleasure across cultures. Proceedings of the National Academy of Sciences, 115(43), E10013-E10021. https://doi:org/10.1073/pnas.1807862115

Chung Y, Rabe-Hesketh S, Dorie V, Gelman A, Liu J (2013). A nondegenerate penalized likelihood estimator for variance parameters in multilevel models. Psychometrika, 78(4), 685-709. https://gllamm.org/

Cordaro, D. T., Sun, R., Keltner, D., Kamble, S., Huddar, N., \& McNeil, G. (2018). Universals and cultural variations in 22 emotional expressions across five cultures. Emotion, 18(1), 75-93. https://doi.org/10.1037/emo0000302.

Du, S., Tao, Y., \& Martinez, A. M. (2014). Compound facial expressions of emotion. Proceedings of the National Academy of Sciences, 111(15), E1454-E1462. https://doi.org/10.1073/pnas.1322355111

Ekman, P. (1992). An argument for basic emotions. Cognition and Emotion, 6(3-4), 169-200. https://doi.org/10.1080/02699939208411068

Ekman, P., \& Cordaro, D. (2011). What is meant by calling emotions basic. Emotion Review, 3(4), 364-370. https://doi.org/10.1177/1754073911410740

Ekman, P., Friesen, W.V., \& Hager, J. V. (2002). Facial action coding system (2 ${ }^{\text {nd }}$ ed.). Research Nexus eBook.

Elfenbein, H. A., \& Ambady, N. (2002). On the universality and cultural specificity of emotion recognition: a meta-analysis. Psychological Bulletin, 128(2), 203-235. https://doi.org/10.1037/0033-2909.128.2.203 
Elfenbein, H. A., Beaupré, M., Lévesque, M., \& Hess, U. (2007). Toward a dialect theory: cultural differences in the expression and recognition of posed facial expressions. Emotion, 7(1), 131-146. https://doi.org/10.1037/1528-3542.7.1.131

Fang, X., Sauter, D. A., \& Van Kleef, G. A. (2018). Seeing mixed emotions: The specificity of emotion perception from static and dynamic facial expressions across cultures. Journal of Cross-Cultural Psychology, 49(1), 130-148. https://doi.org/10.1177/0022022117736270

Fang, X., Van Kleef, G. A., \& Sauter, D. A. (2019). Revisiting cultural differences in emotion perception between easterners and westerners: Chinese perceivers are accurate, but see additional non-intended emotions in negative facial expressions. Journal of Experimental Social Psychology, 82, 152-159. https://doi.org/10.1016/j.jesp.2019.02.003

Freedman, D. G. (1964). Smiling in blind infants and the issue of innate versus acquired. Journal of Child Psychology and Psychiatry, 5(3-4), 171-184. https://doi.org/10.1111/j.1469-7610.1964.tb02139.x

Galati, D., Scherer, K. R., \& Ricci-Bitti, P. E. (1997). Voluntary facial expression of emotion: Comparing congenitally blind with normally sighted encoders. Journal of Personality and Social Psychology, 73(6), 1363. https://doi.org/10.1037/0022-3514.73.6.1363

Giner-Sorolla, R., Kupfer, T., \& Sabo, J. (2018). What makes moral disgust special? An integrative functional review. In Advances in experimental social psychology (Vol. 57, pp. 223-289). Academic Press.

Gudykunst, W. B., Matsumoto, Y., Ting-Toomey, S., Nishida, T., Kim, K., \& Heyman, S. (1996). The influence of cultural individualism-collectivism, self construals, and individual values on communication styles across cultures. Human Communication Research, 22(4), 510-543. https://doi.org/10.1111/j.1468-2958.1996.tb00377.x 
Gudykunst, W. B., \& Ting-Toomey, S. (1988). Culture and affective communication. American Behavioral Scientist, 31(3), 384-400.

https://doi.org/10.1177/000276488031003009

Haidt, J., \& Keltner, D. (1999). Culture and facial expression: Open-ended methods find more expressions and a gradient of recognition. Cognition \& Emotion, 13(3), 225-266. https://doi.org/10.1080/026999399379267

Hall ET (1976). Beyond Culture. Anchor Books/Doubleday.

Hess, U., Kafetsios, K., Mauersberger, H., Blaison, C., \& Kessler, C. L. (2016). Signal and noise in the perception of facial emotion expressions: From labs to life. Personality and Social Psychology Bulletin, 42(8), 1092-1110. https://doi.org/10.1177/0146167216651851

Hunt, W. A. (1941). Recent developments in the field of emotion. Psychological Bulletin, 38(5), 249-276. https://doi.org/10.1037/h0054615

Izard, C. E. (2007). Basic emotions, natural kinds, emotion schemas, and a new paradigm. Perspectives on Psychological Science, 2(3), 260-280. https://doi.org/10.1111/j.17456916.2007.00044.x

Jack, R. E., Sun, W., Delis, I., Garrod, O. G., \& Schyns, P. G. (2016). Four not six: Revealing culturally common facial expressions of emotion. Journal of Experimental Psychology: General, 145(6), 708-730. https://doi.org/10.1037/xge0000162

Kooperberg, C., \& Ruczinski, I. (2018). LogicReg: Logic Regression (version 1.5.10). Obtained from: https://CRAN.R-project.org/package=LogicReg

Krumhuber, E. G., Kappas, A., \& Manstead, A. S. (2013). Effects of dynamic aspects of facial expressions: A review. Emotion Review, 5(1), 41-46. https://doi.org/10.1177/1754073912451349 
Krumhuber, E. G., Küster, D., Namba, S., Shah, D., \& Calvo, M. G. (2019). Emotion recognition from posed and spontaneous dynamic expressions: Human observers versus machine analysis. Emotion. https://doi.org/10.1037/emo0000712

Lucas, G. M., Gratch, J., King, A., \& Morency, L. P. (2014). It's only a computer: Virtual humans increase willingness to disclose. Computers in Human Behavior, 37, 94-100. https://doi.org/10.1016/j.chb.2014.04.043

Matsumoto, D., \& Hwang, H. S. (2012). Culture and emotion: The integration of biological and cultural contributions. Journal of Cross-Cultural Psychology, 43(1), 91-118. https://doi.org/10.1177/0022022111420147

Matsumoto, D., Olide, A., Schug, J., Willingham, B., \& Callan, M. (2009). Cross-cultural judgments of spontaneous facial expressions of emotion. Journal of Nonverbal Behavior, 33(4), 213-228. https://doi.org/10.1007/s10919-009-0071-4

Matsumoto, D., Olide, A., \& Willingham, B. (2009). Is there an ingroup advantage in recognizing spontaneously expressed emotions?. Journal of Nonverbal Behavior, 33(3), 181-191. https://doi.org/10.1007/s10919-009-0068-z

Matsumoto, D., Yoo, S. H., Fontaine, J., Anguas-Wong, A. M., Ariola, M., Ataca, B.,...Grossi, E. (2008). Mapping expressive differences around the world: The relationship between emotional display rules and individualism versus collectivism. Journal of Cross-Cultural Psychology, 39(1), 55-74. https://doi.org/10.1177/0022022107311854

Matsumoto, D., Yoo, S. H., Hirayama, S., \& Petrova, G. (2005). Development and validation of a measure of display rule knowledge: the display rule assessment inventory. Emotion, 5(1), 23-40. https://doi.org/10.1037/1528-3542.5.1.23

Mesquita, B., \& Frijda, N. H. (1992). Cultural variations in emotions: a review. Psychological Bulletin, 112(2), 179-204. https://doi.org/10.1037/0033-2909.112.2.179 
Naab, P. J., \& Russell, J. A. (2007). Judgments of emotion from spontaneous facial expressions of New Guineans. Emotion, 7(4), 736-744. https://doi.org/10.1037/15283542.7.4.736

Nelson, N. L., \& Russell, J. A. (2013). Universality revisited. Emotion Review, 5(1), 8-15. https://doi.org/10.1177/1754073912457227

Niedenthal, P. M., Rychlowska, M., Zhao, F., \& Wood, A. (2019). Historical Migration Patterns Shape Contemporary Cultures of Emotion. Perspectives on Psychological Science, 14(4), 560-573. https://doi.org/10.1177/1745691619849591

Rinn, W. E. (1991). Neuropsychology of facial expression. In R. Feldman \& B. Rime (Eds.), Fundamentals of nonverbal behavior (pp. 3-70). Cambridge University Press.

Rychlowska, M., Miyamoto, Y., Matsumoto, D., Hess, U., Gilboa-Schechtman, E., \& Niedenthal, P. M. (2015). Heterogeneity of long-history migration explains cultural differences in reports of emotional expressivity and the functions of smiles. Proceedings of the National Academy of Sciences, 112(19), E2429-E2436. https://doi.org/10.1073/pnas.1413661112

Ruczinski, I., Kooperberg, C., \& LeBlanc, M. (2003). Logic regression. Journal of Computational and Graphical Statistics, 12(3), 475-511. https://doi.org/10.1198/1061860032238

Sauter, D. A., Eisner, F., Ekman, P., \& Scott, S. K. (2010). Cross-cultural recognition of basic emotions through nonverbal emotional vocalizations. Proceedings of the National Academy of Sciences, 107(6), 2408-2412. https://doi.org/10.1073/pnas.0908239106

Schaefer, A., Nils, F., Sanchez, X., \& Philippot, P. (2010). Assessing the effectiveness of a large database of emotion-eliciting films: A new tool for emotion researchers. Cognition and Emotion, 24(7), 1153-1172. https://doi.org/10.1080/02699930903274322 
Scherer, K. R., Clark-Polner, E., \& Mortillaro, M. (2011). In the eye of the beholder? Universality and cultural specificity in the expression and perception of emotion. International Journal of Psychology, 46(6), 401-435. https://doi.org/10.1080/00207594.2011.626049

Susskind, J. M., Littlewort, G., Bartlett, M. S., Movellan, J., \& Anderson, A. K. (2007). Human and computer recognition of facial expressions of emotion. Neuropsychologia, 45(1), 152-162. https://doi.org/10.1016/j.neuropsychologia.2006.05.001

Tcherkassof, A., Bollon, T., Dubois, M., Pansu, P., \& Adam, J. M. (2007). Facial expressions of emotions: A methodological contribution to the study of spontaneous and dynamic emotional faces. European Journal of Social Psychology, 37(6), 1325-1345. https://doi.org/10.1002/ejsp.427

Thompson, J. (1941). Development of facial expression of emotion in blind and seeing children. Archives of Psychology, 37, 1-47. https://psycnet.apa.org/record/1942-00976001

Tsai, J. L., \& Chentsova-Dutton, U. (2003). Variation among European Americans in emotional facial expression. Journal of Cross-Cultural Psychology, 34(6), 650-657. https://doi.org/10.1177/0022022103256846

Tsai, J. L., Ang, J. Y. Z., Blevins, E., Goernandt, J., Fung, H. H., Jiang, D., ... \& Lin, Y. (2016). Leaders' smiles reflect cultural differences in ideal affect. Emotion, 16(2), 183195. https://doi.org/10.1037/emo0000133

Van Kleef, G. A. (2016). The interpersonal dynamics of emotion: Toward an integrative theory of emotions as social information. Cambridge University Press.

Wood, A., Rychlowska, M., \& Niedenthal, P. M. (2016). Heterogeneity of long-history migration predicts emotion recognition accuracy. Emotion, 16(4), 413-420. https://doi.org/10.1037/emo0000137 